\title{
Affinity Maturation of the Humoral Immune Response: A Bayesian Approach
}

\author{
Sujit Kumar GHOSH \\ Department of Statistics, North Carolina State University \\ Institute of Statistics Mimeo Series No. 2532 \\ May 2001
}

\begin{abstract}
We consider statistical modelling of a simplified version of Darwinian evolution which involves only two genes encoding one molecule. In particular, we consider a process known as affinity maturation that is carried out in Germinal Center Reaction (GCR). We use a statistical model developed in Radmacher et al (1998) that enables us to tease apart the effects of mutation rate and selection in B cell populations undergoing an immune response. Standard classical statistical approaches that uses large-sample theory may lead to misleading conclusions, when sample size is very small. The data collection in many biological systems, including the immune response of vertebrate organisms, is both time consuming and often results in relatively small data sets. We therefore, take a Bayesian perspective that produces finite-sample estimates of the mutation rates and is flexible in extending the models to more complicated process. We illustrate our method using a sequence data obtained from Gen bank that carried the experiment over two years.
\end{abstract}


Key words and phrases. Bayesian hierarchical models, Biological sequence data, Germinal Center Reaction, MCMC methods, WinBUGS. 


\section{Introduction}

Evolution by variation and selection has become the starting point for all of biology (see Darwin, 1859). Genetic changes are generated at random and are subsequently subject to selection. Those changes that confer upon their bearers a reproductive advantage propagate through the population and may even displace the ancestral type. This fundamental process underlies both large-scale events, such as speciation (see, e.g., Futuyma (1997) and smaller-scale events, such as fine-tuning of the genotype in fluctuating environments as beautifully documented for the Galapagos finches by Rosemary and Peter Grant in Grant and Grant (1989). A particularly remarkable, and somewhat surprising, example of small-scale and rapid evolution is that of the immunoglobulin molecules during the immune response. The process is known as affinity maturation (reviewed in Berek, 1999) and is carried out in the Germinal Center Reaction $(G C R$ ) (see Jacob et al., 1991) and Berek et al., 1991). This process, which we will outline below, is a considerably simplified instance of Darwinian evolution, involving just two genes encoding one molecule, but is nevertheless surprisingly complex.

The GCR presents interesting opportunities and challenges to applied statistics. Radmacher et al. (1998) analyzed data from a series of experiments and concluded that the selective process is more complex than previously believed and shows a strong stochastic component.

Radmacher and Kepler (1999) followed up with a closer look, at the statistical properties of the model and used extensive simulations to draw their inferences. The methodology they employed was likelihood-based, and among the conclusions drawn from this latter work was the indication that inference based on large-sample properties can be misleading, when the sample size is as small as 16-20 observations.

We have conducted a Bayesian analysis of the data, and present here the 
resolution of several issues of concern for their biological or statistical assumptions and implications. In Section 2, we describe the GCR from biological considerations. In Section 3, we formulate the statistical model for the GCR data. In the two subsections of Section 3 , we briefly discuss the classical statistical methods of estimation and point out its limitations for our data. We then propose a Bayesian approach that overcomes some of the limitations of the classical approach. Section 4, provides specific details on how to fit the Bayesian model using Markov Chain Monte Carlo (MCMC) with the Bayesian software WinBUGS. In Section 5, we use WinBUGS to analyze the sequence data. Finally in Section 6, we make some general conclusions and indicate sources of future research.

\section{The GCR model}

We provide a brief description of both the biology of the GCR and of the mathematical model underlying the statistical model used here. The interested reader is encouraged to seek further details in Berek (1998) for the biology and in Radmacher et al (1998) for the modelling.

Within two or three days after immunization, a subset of the pathogenspecific B cells that have been activated as part of the immune response migrate to specialized areas of the lymph nodes and spleen where, along with $\mathrm{T}$ lymphocytes and follicular dendritic cells, they form structures known as germinal centers. Here, the B cells become centroblasts and cycle very rapidly; the cell-cycle time is estimated to be 6-7 hours (see Holloway and McCartney, 1992). In addition, the DNA encoding in the variable regions of the heavy and light chains undergo hypermutation and are subjected to mutation rates estimated to be $10^{-3}$ substitutions per nucleotide per cell division as observed by McKean et al (1984) and Clarke et al (1985). This rate is sufficient to produce, on average, one mutated daughter cell per division. 
In order to continue proliferating, the B cells must receive signals from other cells in the germinal center. They migrate to another zone of the germinal center where they encounter molecules from the eliciting pathogen sequestered on follicular dendritic cells, cells specialized for the task of "presenting" such molecules to germinal center B cells. B cells whose immunoglobulin molecules have sufficient affinity for these pathogen molecules are receive survival signals in this interaction. Having been so signaled, they must now get another signal from T cells in the germinal center (see Han et al, 1995) after which they may continue to proliferate.

Because one signal for survival requires binding of the cell's immunoglobulin molecules to molecules from the eliciting pathogen, those cells that, by acquiring mutations in their variable-region genes, have increased the affinity of this interaction, have a selective advantage and will increase their representation in the population. Thus the immune response gain specificity and high affinity. Some of the cells that pass through the germinal center successfully go on to become memory cells and maintain the immune memory for this specific pathogen. The second exposure to the same pathogen is then handled much more rapidly and efficiently than the first.

Our aim here is to take a closer look at the roles of mutation and selection in this process and to show how Bayesian methodology can aid in determining, how the mutation and selection individually contribute to the overall time required to complete the reaction. To this end we have analyzed data acquired by the Garnett Kelsoe immunology lab (for further details on the experimental procedure, see Jacob et al, 1991). This data are from mice immunized with the hapten NP conjugated with chicken gamma globulin. A crucial feature of this system, shared with several other experimental hapten systems, is that there is a recurring point mutation, referred to as a "key" mutation, which confers a ten-fold increase in affinity and is found to dominate the response at late times (see Allen et al, 1988). This allows us to classify the variable region sequences 
that are recovered in the experiment dichotomously, as key or non-key.

The data in Table 1, consist of variable region gene sequences recovered from different mice at different times after immunization. Data were obtained from studies of the immune response against (4-hydroxy-3-nitrophenyle) acetyl (NP) in C57BL16 mice. In this system a tryptophan-to-lecucine exchange at position 33 in complementarity determining region 1 (CDR1) commonly appears in secondary response antibodies encoded by the $V_{H}$ 186-2 gene. The position 33 mutation, a transversion from $\mathrm{G}$ to $\mathrm{T}$ in the second nucleutide of the codon, produces a 10-fold increase in binding affinity of the $I_{g}$ for NP. Our task is to estimate the effective mutation rate and the time to dominance once the key mutation is seeded. The model we have adopted for this purpose simplifies the situation by breaking the eventual succession of key mutants into two stages. During the first stage, there are no key mutants but they are potentially being generated by mutations from non-key cells. In the second stage, the key cells have been established and the clone of key cells grows by proliferation. The first stage is modelled as a Poisson process, while the second is modelled as a deterministic population growth model with random initial time inherited from the first stage (see Radmacher et al, 1998 for more details). An implicit assumption made here is that the variability associated with the proliferation phase following the seeding of the key mutation is negligible compared to the variability associated with the seeding process itself.

The specific question we are interested in addressing is whether or not the estimated Poisson intensity associated with the seeding process is consistent with the estimated rate found by other means McKean et al (1984) and Clarke et al (1985). If it is not, it suggests that the two rates are not the same; that there is an additional process involved in seeding that is not involved with the generation of mutants, per se. In particular, it might be the case, that there is a stochastic component of selection that acts along with the strictly affinitybased component. In other words, even cells that do bear the high-affinity 
key mutations might, by luck of the draw, fail to get proliferation signals by being denied access to the network of follicular dendritic cells or to the right T cells. In either case, key cells would be produced but fail to produce clones; the Poisson intensity estimate based on the time delay to appearance of clones would then not be consistent with that derived from the basal mutation rate itself.

\section{A Statistical Model for GCR}

In developing the statistical model for the biological process described in Section 2, we introduce the following notations for the ease of understanding. For $i=1,2, \ldots, n=16$, let,

$m_{i}=$ total number of Ig sequences sampled from $\mathrm{GC} i$,

$X_{i}=$ number of position 33 mutations from GC $i$ (out of $m_{i}$ ) taken from GC $i$ at the time $t_{i}$,

$\rho_{i}=$ proportion of key cells in GC $i$, and

$\tau_{i}^{\star}=$ time of appearance of the founder in $\mathrm{GC} i$.

Of the 16 GC samples that constitute the data set in Table 1, only five contain key mutations. Thus although it is predicted that on average, a key mutant appears in nearly every generation, these mutants are not being perpetuated in most of the GC.

The statistical model is specified in two stages. At the first stage, there are no key mutants, however we assume that the key mutants are potentially being generated from mutations from non-key cells according to a Poisson process

with constant intensity $\lambda=1 / \tau$. In other words, the times $\tau_{i}^{\star}$ for the arrival of the founder mutant are modelled as independent identically distributed exponential random variables with constant mean $\tau$. 
In the second stage (after the appearance of the founder) the founder key is selected to proliferate and eventually dominate a GC. Hence, we model the growth of proportion $\rho_{i}$, of key mutants among the cells in the $i$ th GC. We assume a deterministic logistic growth curve with growth rate $k$ :

$$
\frac{d \rho_{i}}{d t_{i}}=k \rho_{i}\left(1-\rho_{i}\right) \text {, for } t_{i}>\tau_{i}^{*},
$$

where the initial condition of the differential equation, $\rho_{i}(0)=\rho_{0}$ is the proportion of key mutants among all the dividing cells in the germinal center at the time of the founder's appearance. This is clearly of order $1 / N$ where $N$ is the total number of dividing cells at the time of the founder's appearance. The value $\rho_{0}$ used previously (Radmacher \& Kepler, 1998) was based on an estimate of this $N$. Our method recognizes the uncertainty in this estimate and allows $\rho_{0}$ to be an unknown parameter in the model.

The solution to the differential equation (1) is given by

$$
\rho_{i}=\rho\left(k, \tau_{i}^{\star}, \alpha\right)=\left[1+\exp \left\{\alpha-k\left(t_{i}-\tau_{i}^{\star}\right)\right\}\right]^{-1} \text { for } t_{i} \geq \tau_{i}^{*}
$$

where $\alpha=\log \left(\frac{1-\rho_{0}}{\rho_{0}}\right)$.

In order to derive the likelihood function we combine the two-stages into a single model. This is achieved using Binomial sampling. In other words, the conditional distribution of $X_{i}$ given $\tau_{i}^{\star}$ can be obtained as a zero-inflated Binomial distribution (see Ghosh et al, 1999). More precisely,

$$
P\left(X_{i}=x_{i} \mid \tau_{i}^{\star}\right)= \begin{cases}\left(1-\theta_{i}\right)\left(1-\rho_{i}\right)^{m_{i}}+\theta_{i} & \text { if } x_{i}=0, \\
\left(1-\theta_{i}\right)\left(\begin{array}{c}
m_{i} \\
x_{i}
\end{array}\right) \rho_{i}^{x_{i}}\left(1-\rho_{i}\right)^{m_{i}-x_{i}} & \text { if } x_{i} \neq 0 .\end{cases}
$$

where $\theta_{i}=\exp \left(-\lambda t_{i}\right)$ is the probability that no founder key mutant has arrived up to time $t_{i}$.

To complete the model specification we need to specify the marginal distribution of the appearance time, $\tau_{i}^{\star}$ 's. The marginal distribution of the random times $\tau_{i}^{\star}$ are assumed to be independently distributed as truncated exponential 
distribution. More precisely,

$$
f\left(\tau_{i}^{\star} \mid \lambda\right)= \begin{cases}\frac{\lambda \exp \left(-\lambda \tau_{i}^{\star}\right)}{1-\exp \left(-\lambda t_{i}\right)} & \text { if } 0 \leq \tau_{i}^{\star} \leq t_{i}, \\ 0 & \text { otherwise }\end{cases}
$$

Since we are not able to observe the $\tau_{i}^{\star}$ 's, the likelihood based on the observed data is the marginal distribution of $X_{i}$ (given the parameters $k, \lambda$ and $\alpha$ ). This is obtained by integrating out $\tau_{i}^{\star}$ over the interval $\left[0, t_{i}\right]$. In other words,

$$
P\left(X_{i}=x_{i} \mid k, \lambda, \alpha\right)=\int_{0}^{t_{i}} P\left(X_{i}=x_{i}\right) f\left(\tau_{i}^{\star} \mid \lambda\right) d \tau_{i}^{\star} .
$$

The likelihood function of the parameters $(k, \lambda, \alpha)$ are obtained by multiplying the above probabilities over $i=1, \ldots, n$.

$$
\mathcal{L}(k, \lambda, \alpha)=\prod_{i=1}^{n} P\left(X_{i}=x_{i} \mid k, \lambda, \alpha\right) .
$$

Notice that the explicit (analytical) evaluation of the above likelihood function is not possible. Numerical integrations are required to evaluate the integrals involved for a given set of parameter values. From now on we will denote the set of parameters by $\theta=(k, \lambda, \alpha)$ and the data by $X=\left(x_{1}, \ldots, x_{n}\right)$. One of main contributions of this article is to show that direct numerical integrations are not required to obtain the parameter estimates.

\subsection{Classical methods of estimation and its limitations}

Using traditional statistical methods, we usually estimate the parameters, by maximizing the likelihood in (3). The maximizer or the mode $\hat{\theta}=(\hat{k}, \hat{\lambda}, \hat{\alpha})$, is called the maximum likelihood estimator (MLE). However, such optimizations are not straightforward for our model. Numerical recipes are required for such optimizations (see Radmacher et al, 1998). Other sophisticated iterative techniques like $E M$ algorithm can also be applied.

A point estimate, is not particularly useful unless there is also some measure of uncertainty associated with it. Thus, even if, we successfully optimize 
the above function (or the logarithm of the function) using some efficient (derivative-free) techniques (such as Powell's algorithm or EM algorithm), the main difficulty is to obtain a measure of uncertainty of the point estimates based on only $n=16$ observations. Use of large-sample theory can be misleading with such a small sample size.

A stochastic version of the $E M$ algorithm produces estimates of the variancecovariance matrix of the distribution of the MLE, however the properties of such estimates relies on asymptotic theory. In other words, the standard error of the MLE's are usually not computable for finite samples. Even when, the regularity conditions for large-sample theory are true, the large-sample distributions of the MLE's could be very different from their corresponding small-sample distributions.

With lot of effort scientists were able to obtain only $n=16$ observations for our data, over a period of two years. So in order to make statistical inference we can not rely on the large-sample theory. Specifically, for our model, these limitations of classical methods, along with other difficulties, are discussed in Radmacher and Kepler (1999), based on extensive simulations. In particular using the Kolmogorov-Smirnov (KS)-test, the authors show that the Monte Carlo distributions of the MLEs differ greatly from their respective asymptotic distributions. The authors also point out that different parameterizations (say for $\lambda=1 / \tau$ ) lead to dramatically different (large-sample) confidence intervals. Thus, one stumbling block in the process is the relative scarcity of data available for many cellular and molecular phenomena. In the next section we propose a Bayesian method based on the small data set, to draw conclusions from the analysis of our model that have biological significance.

\subsection{The Bayesian Paradigm}

In order to avoid some of the above mentioned problems, we take a different route to making statistical conclusions. We adopt a method which is known 
as Bayesian inference. The main idea of this methodology, is to consider a full probability model for all observables (response, covariates etc.) and unobservables (parameters, missing data etc.). This full probability models is specified in two stages. First, a probability distribution known as, prior distribution is considered for all unobservables such as parameters $\tau, k$ and $\alpha$. Then a sampling distribution is proposed for the conditional distribution of observables (such as data $x_{i}$ ) given the unobservables. Then using the Bayes' Rule, the prior information is updated to posterior distribution, the conditional distribution of the parameters given the data that is actually observed.

This approach allows us to bring in biological expertise into our model by incorporating the scientist's (prior) belief. Then in the light of data, prior belief is updated to posterior distribution. This framework provides a unified theory of making statistical inference and provides finite-sample estimates in contrast to large sample theory.

For our model, we use informative prior distribution, specified by biological considerations. However we keep the prior uncertainty large, so as to keep our inference similar to maximum likelihood theory. Other informative (subjective) prior distributions can be used if such information becomes available.

Prior distributions for our model can be elicited in several ways. We take the simplest route by restricting the range of the parameters and using flat (uniform) distributions on that range. For example, from biological considerations when we know that the growth rate, $k$ can not exceed say 4 , then we could say that $k$ has a uniform distribution over $(0,4)$. However, if we have more knowledge about the parameter $k$, we could consider other probability distributions with support $(0,4)$. Similarly, existing biological considerations lead us to believe that the parameter $\lambda$ can not exceed the number unity, so for $\lambda$, we consider an uniform distribution on $(0,1)$. Finally, for the parameter $\alpha$, we know that $\rho_{0}=1 / 2300$ is a potential value. In fact this value was used, as a fixed number in Radmacher and Kepler (1999). On the logarithmic scale which 
implies $\alpha \approx 7.74$. Thus, if we do not have any other information, we could consider an uniform distribution on say $(6,9)$. Another possibility is to use a normal distribution for $\alpha$ with mean 7.74 and some large standard deviation. Our code (see Appendix) can easily incorporate such possibilities.

Thus, taking into account the above considerations, we use the following prior distributions for our model,

$$
\begin{aligned}
k & \sim U(0,4), \\
\lambda & \sim U(0,1) \\
\text { and } \alpha & \sim U(6,9) .
\end{aligned}
$$

Assuming prior independence of $k, \lambda$ and $\alpha$, we obtain the joint prior distribution,

$$
p(k, \lambda, \alpha)=\frac{1}{12} \text { where } 0 \leq k \leq 4,0 \leq \lambda \leq 1,6 \leq \alpha \leq 9 .
$$

The above probability statements represents our prior belief based on only range considerations. Other probability statements, that express our prior uncertainty, could be easily incorporated if such information become available. Notice that prior independence doesn't necessarily imply that $k, \lambda$ and $\alpha$ are independent it aposteriori.

\section{Parameter Estimation using MCMC}

For our model the sampling distribution is given by the likelihood function $\mathcal{L}(k, \lambda, \alpha)$ in $(3)$ and the prior is given in (4). The posterior distribution of $\theta=(k, \lambda, \alpha)$ is obtained via the Bayes' Rule:

$$
\begin{aligned}
p(\theta \mid X) & =\frac{\mathcal{L}(\theta) p(\theta)}{\int p(\theta) d \theta} \\
& \propto \mathcal{L}(\theta) p(\theta)
\end{aligned}
$$

From above it appears that finding the posterior distribution could be more troublesome than the traditional methods, because of the high-dimensional in- 
tegration. However, with the development of computer-based statistical methods, a relatively new collection of tools called the Markov Chain Monte Carlo (MCMC) has made such problems rather easy (see Tierney, 1994). The main idea of MCMC is to construct a Markov Chain with the posterior distribution (i.e., $p(\theta \mid X))$ as its stationary distribution. This is done using a simple strategy developed by Metropolis in mid 50's and later generalized by Hastings in early 70's (for a review see Chib and Greenberg, 1995). One advantage of this strategy is that it does not involve the calculation of the normalizing constants of the posterior distributions. Now a days a software known as WinBUGS can implement such strategies quite easily for not-so-complicated models. However a lot of care must be taken to ensure the convergence of such Markov Chains (MC). We use the version 1.3 of WinBUGS (that comes with G-R for convergence diagnostic) to fit our Bayesian model. See the Appendix on how to obtain the code we used. Minor alterations are required to modify or change the prior distributions or their hyper-parameters. In particular, the likelihood in (3) can be specified as a hierarchical Bayes model,

$$
\begin{aligned}
X_{i} \mid b_{i}, \rho_{i} & \sim \operatorname{Binomial}\left(\rho_{i}\left(1-b_{i}\right), m_{i}\right) \\
b_{i} \mid \lambda & \sim \operatorname{Bernoulli}\left(\exp \left\{-\lambda t_{i}\right\}\right) \\
\rho_{i} & =\left[1+\exp \left\{\alpha-k\left(t_{i}-\tau_{i}^{\star}\right)\right\}\right]^{-1} \\
\tau_{i}^{\star} & \sim \text { Exponential }(\lambda) I\left(0, t_{i}\right)
\end{aligned}
$$

where Exponential $(\lambda) I\left(0, t_{i}\right)$, denotes an Exponential distribution with mean $\tau=1 / \lambda$ right truncated at $t_{i}$. The hierarchical version in of the likelihood in (5) together with the prior as given in (4) can be used to fit models in WinBUGS (see Appendix for the code).

In a Bayesian model, all relevant statistical inferences about the parameters are based on the posterior distribution of the parameter(s). e.g. an equal tail $95 \%$ credible interval (similar to confidence interval) is obtained by finding the 2.5 and 97.5 percentiles of the posterior distribution. Such a distribution facil- 
itates a common-sense interpretation of statistical conclusion. For instance, a Bayesian credible interval for an unknown parameter of interest can be regarded as having high probability of containing the unknown parameter, in contrast to frequentist confidence interval, which may strictly be interpreted only in relation to a sequence of similar inferences that might be made in repeated trials. A Bayesian credible interval is computed quite easily using sampling-based methods such as MCMC. This is another advantage of MCMC; most of the summary values of the posterior distribution is obtained from MCMC sample using its sample analogue. The accuracy of such sample analogue is judged using the Ergodic Theorem of Markov Chains.

More precisely, if $\theta^{(1)}, \theta^{(2)}, \ldots, \theta^{(m)}, \ldots$ are realizations of a Markov Chain with stationary distribution $p(\theta \mid X)$, then after some "burn-in time" say $m_{0}$, the observations $\theta^{\left(m_{0}\right)}, \theta^{\left(m_{0}+1\right)}, \ldots$, can be regarded as realizations from the desired posterior distribution $p(\theta \mid X)$. Hence any summary value say $E[g(\theta) \mid X]$ can be approximated (up to only Monte Carlo error), by

$$
E[g(\theta) \mid X] \approx \frac{1}{M} \sum_{i=m_{0}}^{m_{0}+M-1} g\left(\theta^{(i)}\right) .
$$

where $g(\cdot)$ is some real-valued function of $\theta$.

Notice that $M$ is not the sample size $n$. So given the computer capability and time, we can use as large $M$ as we desire, to reduce the Monte Carlo error. In practice, some initial iteration (usually $10 \%$ of all samples to be generated) generated from a Markov chain is discarded to allow sufficient "burn-in" and $N=5000$ samples are kept for making statistical inference based on posterior distribution. However such guidelines are truly problem-dependent and should not be taken as recommendations. In particular, for our model-fitting we generated 15, 000 samples, then burned-in $m_{0}=5000$ samples and finally retained every 10th sample for a total of $M=1000$ samples for each parameter. The trace plots and posterior densities of $k, \lambda$ and $\alpha$ are presented in Figure 1.

For our models, on a Sparc-20 station it took only few seconds to run the 
whole chain. There are some standard guidelines to detect the burn-in time and hence the convergence of the MC. Among others diagnostic techniques, most widely used techniques are by Gelman-Rubin (G-R) based on overdispersed starting values (see Gelman and Rubin, 1990) and Raftery-Lewis, based on a single chain (see Raftery and Lewis, 1995). A review of currently available convergence diagnostics can found in Carlin and Cowles (1997). We used both of this methods along with some graphical techniques (such as trace plots, ACF plots) to check if our chains have any problem with convergence. All these plots and numerical diagnostics are obtained using the S-plus package CODA. We provide more details on the Bayesian output analysis of sequence data in the next section.

\section{Data Analysis using WinBUGS}

We obtained the data for our study from Radmacher et al (1998). The data has been reproduced in Table 1. For the source and description the data see Section 2. After some preliminary runs, we ran our code (see Appendix) in WinBUGS for a total of 15,000 iterations, discarded initial 5000 iterations. After we obtain the output from WinBUGS we transferred the output into CODA for Bayesian output analysis.

First, to check convergence of the MCMC sampler, we used Geweke's diagnostic checks to see if there is any problem with convergence. In Figure 2 we present Geweke's diagnostics plots, which clearly indicates that there is no serious problem of convergence. For more details Geweke's convergence criteria, the reader is referred to CODA manual. The number 5000 for burn-in was chosen (arbitrarily) large to allow sufficient time so that the samples start coming from the stationary distribution i.e. the posterior distribution of $\theta$ given the data $X$. From our CODA output we observed that lag-10 autocorrelations of $\alpha, k$ 
and $\lambda$ were $0.10,0.20$ and 0.03 respectively compared to lag- 1 autocorrelation of $0.79,0.88$ and 0.35 . This can be observed in Figure 3 which plots the autocorrelation function (ACF) of the 10,000 samples after initial 5000 burn-ins for each parameter. So we decided to keep every 10-th iteration to obtain approximately i.i.d sample from the posterior distribution. Figure 4 gives the plot of autocorrelations of the final 1000 samples (based on keeping every 10th from the 10,000 samples). We clearly see that the autocorrelations dies off almost immediately. We also used multiple dispersed starting values for the MCMC chain and checked convergence using other available numerical tests in CODA, (not presented here) such as Gelman-Rubin, Raftery Lewis, Heidelberg-Welch etc., all of which indicated no problem with the convergence of the MCMC sampler.

The posterior summary values based on the MCMC output is presented in Table 2. The summary values are based on the final 1000 samples. To compare our results with that obtained by Radmacher et al (1998) we fitted two Bayesian models; Model-I assumes that $\alpha$ is unknown and Model-II assumes that $\alpha=7.74$ is fixed. For Model-I we tried several prior distributions for the parameter $\theta$ such as uniforms, log-normals and truncated normals. However the results seemed to be not sensitive to such choices, as long as we kept the priors distribution relatively non-informative with respect to the likelihood.

In Table 2 , we present posterior median along with a $95 \%$ equal-tail credible interval (i.e. $2.5 \%$ and $97.5 \%$ posterior percentiles) for each parameter of interest under Model-I and Model-II. For the corresponding classical model we report the MLE estimate as obtained from the Radmacher et al (1998) article. Clearly Bayesian intervals for $\lambda$ are slightly wider compared to classical counterpart. This is because Bayesian intervals are based on the finite-sample estimates and hence provides a true measure of uncertainty in parameter estimates. In addition, we notice from Figure 1, that the posterior densities of the parameters are all skewed to the right (posterior means of $k, \lambda$ and $\alpha$ are 2.96, 
0.31 and 7.61 respectively) and hence large-sample normal theory may not be appropriate to obtain $95 \%$ C.I.'s of the parameter estimates. Alternatively, even if we use C.I.'s based on inverting a chi-squared test, we didn't see much improvement. We didn't find the interval estimate of $k$ based on the classical estimate, so we couldn't compare the intervals.

Interestingly enough, the posterior estimate of $\alpha$ turns out to be 7.66 (posterior median) or 7.61 (posterior mean). Thus the common value of $\alpha=7.74$, that is used in the literature from biological considerations, seems to be a very good choice. Our Bayesian method gives a statistical justification of this fact. However, the density plot of $\alpha$ in Figure 1, suggests that $\alpha$ might have a bimodal distribution. Although the posterior interval for $\alpha$ seems to be close to that of the prior support (i.e. $(6,9)$ ), we found similar posterior interval estimate when we varied the prior support to different ranges wider than that of $(6,9)$. Recall that the informative prior with range $(6,9)$ was used from biological considerations.

Notice that there is a striking difference in the estimate of $\lambda$; the MLE of $\lambda$ is not even included in the $95 \%$ intervals obtained via Model-I and ModelII. Moreover, there is just a slight overlap between the classical and Bayesian intervals. Similar remarks applies to the parameter $\tau$. This suggests that the classical estimates based on large-sample theory could be very different from its finite sample estimates when the sample size is relatively small. Evidence of such problems with the classical methods are also reported in Radmacher and Kepler (1999). They used simulation studies to confirm that the asymptotic distribution of the parameters (e.g. $\lambda$ ) are significantly different from that of empirical distribution. These authors also reported strikingly different estimates of $\lambda$ and $\tau$ based on different parameterizations. For Bayesian methods, this poses no additional problems as the posterior distribution of $\tau=1 / \lambda$ can be obtained from the MCMC sample od $\lambda$. The posterior summary of $\tau$ is presented in Table 2. Thus our proposed method avoid such problems by 
obtaining finite sample interval estimates. Finally, an added advantage of the our proposed method is that it can be fitted routinely using freely available softwares (see Appendix).

\section{Conclusion and discussion}

In this article we have used a very simple version of the Darwinian evolution model. However even in such simple set-up the statistical analysis becomes quite challenging. We point out the limitation of the traditional statistical models for such complicated parametric models. Our method shows that by viewing the model as made of two stages can simplify the implementation. Also the most important contribution of this article is to show that such complicated parametric models can be fit quite routinely using the software WinBUGS and hence can be used by end-users who need not be experts in using MCMC methods. Our methods do not rely on the inappropriate asymptotics and provide finite sample measure of uncertainty. We hope that for more complex biological models our techniques can be successfully applied even when small number of observations are available. It is natural to extend these two-stage modelling ideas to multiple stages where there are multiple genes encoding one molecule. However model formulation the such complex system can be very tricky! A Bayesian approach to such generalizations is under study by the author.

\section{Acknowledgements}

The author would like to thank the WinBUGS group for letting us use their software free of charge for our model fitting. The author also thanks Dr. Thomas Kepler for providing many valuable insights for the models and for making the AMHIR data accessible to us. 


\section{Appendix}

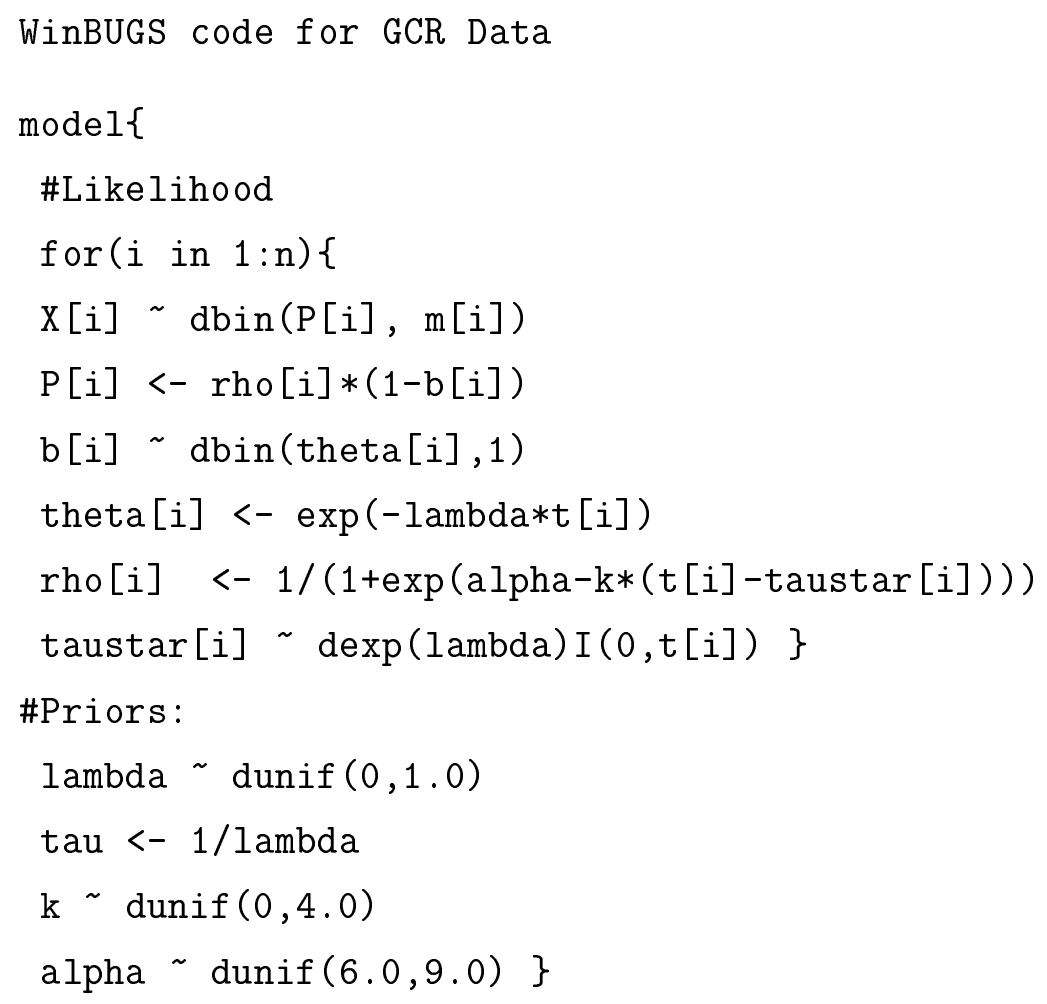

It is the responsibility of the user to download the WinBUGS software from the website http://www.mrc-bsu.cam.ac.uk/bugs/

The software CODA can also be downloaded from the above website. The unix users are recommended to use BUGS instead of the WinBUGS. For more details on implementation and use of the softwares, the reader is requested to visit the WinBUGS website.

\section{References}

Allen, D., Simon, T., Sablitzky, F., Rajewsky, K. and Cumano, A. (1988) Antibody engineering for the analysis of affinity maturation of an antihapten response, EMBO J., 7:1995. 
Berek, C. (1998) Affinity Maturation. In Fundamental Immunology. ed. W.E. Paul, Lippincott, Williams and Wilkins.

Berek, C. (1999) Affinity Maturation. Chapter 25 of Fundamental Immunology, Paul, W.E. ed., Lippincott-Raven, Philadelphia, PA.

Berek, C., Berger, A. and Apel, M. (1991) Maturation of the immune response in germinal centers. Cell, 67, 1121.

Cowles, K. and Carlin, B. P. (1998) Markov Chain Monte Carlo convergence diagnostics: a comparative review. J. Amer. Statist. Assoc., , .

Chib, S. and Greenberg, E. (1995) Understanding the Metropolis-Hasting algorithm. The American Statistician, 49, 327-335.

Clarke, S.H., Huppi K., Ruezinsky, D., Staudt, L., Gerhard, W. and Weigert, M. (1985) Inter- and intraclonal diversity in the antibody response to influenza hemagglutinin. Journal of Experimental Medicine, 161, 687.

Darwin, C. (1859) The Origin of Species : By Means of Natural Selection or the Preservation of Favored Races in the Struggle for Life. Reprinted 1993, Modern Library.

Futuyma, D. J. (1997) Evolutionary Biology, 3rd edition. Sinauer Association.

Gelman, S. and Rubin, D. (1992) Inference from iterative simulation using multiple sequences (with discussion). Statistical Science, 7, 457-511.

Ghosh, S. K., Mukhopadhyay, P. and Lu, J. C. (1999) Bayesian Analysis of Zero-inflated Regression Models, Technical report, NC State University.

Grant, B. R. and Grant, P. R. (1989) Evolutionary Dynamics of a Natural Population: The Large Cactus Finch of the Galapagos. University of Chicago Press, Chicago. 
Han, S., Hathcock, K., Zeng, B., Kepler, T. B., Hodes R. and Kelsoe, G. (1995) Cellular interaction in germinal centers: I. The roles of CD40ligand and B7-2 in established germinal centers. Journal of Immunology, 155, 556-567.

Holloway, K. and McCartney, J. (1992) Cell kinetics of the germinal center reaction - a stathmokinetic study. Eur. J. Immunol. 22-261.

Jacob, J., Kelsoe, G., Rajewsky, K. and Weiss, U. (1991) Intraclonal generation of antibody mutants in germinal centers, Nature, 354-389.

McKean, D., Huppi, K., Bell, M., Staudt, L., Gerhard W. and Weigert, M. (1984) Generation of antibody diversity in the immune response of BALB/c mice to influenza virus hemagglutinin. Proceedings of National Academic Sciences, 81, 3180 .

Radmacher, M. D., Kelsoe, G. and Kepler, T. B. (1998) Predicted and inferred waiting times for key mutations in the germinal center reaction: Evidence for stochasticity in selection. Immunology and Cell Biology, 76, 373-381.

Radmacher, M. D. and Kepler, T. B. (1999) Modelling mutation and selection in the germinal center reaction by maximum likelihood, Technical Report, NC State University.

Raftery, A. E. and Lewis, S. (1992) How many iterations in the Gibbs sampler? In Bayesian Statistics 4, ed. J. M. Bernardo et al, Oxford University press.

Tierney, L. (1994) Markov chains for exploring posterior distributions (with discussion). Annals of Statististics, 22, 1701-1762. 
Table 1: The sequence data used in the analysis*.

\begin{tabular}{cccc}
\hline $\mathrm{GC} i$ & $t_{i}$ & $m_{i}$ & $x_{i}$ \\
\hline 1. & 8 & 8 & 0 \\
2. & 8 & 10 & 0 \\
3. & 8 & 12 & 0 \\
4. & 8 & 12 & 0 \\
5. & 10 & 9 & 0 \\
6. & 10 & 10 & 10 \\
7. & 10 & 3 & 0 \\
8. & 10 & 7 & 0 \\
9. & 10 & 4 & 0 \\
10. & 14 & 12 & 0 \\
11. & 14 & 11 & 11 \\
12. & 14 & 10 & 8 \\
13. & 14 & 11 & 0 \\
14. & 14 & 8 & 3 \\
15. & 14 & 10 & 0 \\
16. & 16 & 8 & 8 \\
\hline & & &
\end{tabular}

* For details see section 2 in the text. Sequences are available from EMBL/Gen Bank/DDBJ under accession numbers DS13953 and X67341-X67391. 
Table 2: Bayesian and classical estimates with $95 \%$ intervals.

\begin{tabular}{c|ccc}
\hline parameters & $\begin{array}{c}\text { Bayesian } \\
\text { Model-I }\end{array}$ & $\begin{array}{c}\text { Bayesian } \\
\text { Model-II }\end{array}$ & $\begin{array}{c}\text { Classical } \\
\text { Model }\end{array}$ \\
\hline$k$ & 3.26 & 3.27 & 3.87 \\
$\lambda$ & $(2.34,3.94)$ & $(2.53,3.95)$ & $\left(\right.$ na* $^{*}$, na $)$ \\
& 0.33 & 0.33 & 0.11 \\
$\tau$ & $(0.18,0.49)$ & $(0.20,0.53)$ & $(0.04,0.26)$ \\
$\alpha$ & 3.02 & 3.01 & 8.8 \\
& $(1.89,4.99)$ & $(1.94,5.00)$ & $(3.9,25.7)$ \\
& 7.66 & known & known \\
& $(6.11,8.93)$ & $(-,-)$ & $(-,-)$ \\
\hline
\end{tabular}

${ }^{*}$ na: Not available; in the literature. Bayesian Model-II and classical model assumes $\alpha=$ 7.74 . 


\section{Bayesian Output Analysis of Sequence Data}
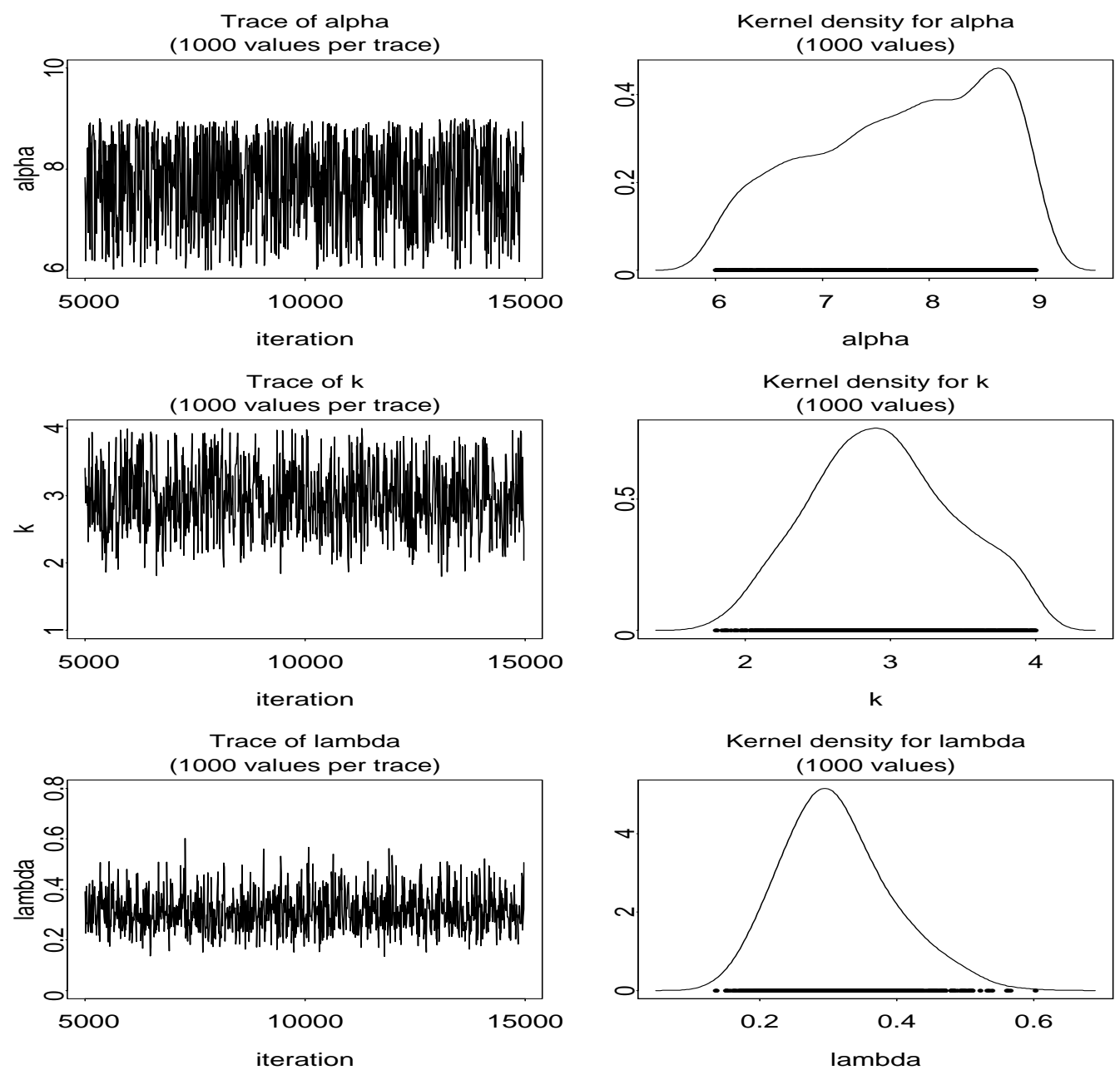

Figure 1: Trace plots and posterior density plots of the parameters. 


\section{Bayesian Output Analysis of Sequence Data}

Geweke's Convergence Diagnostic

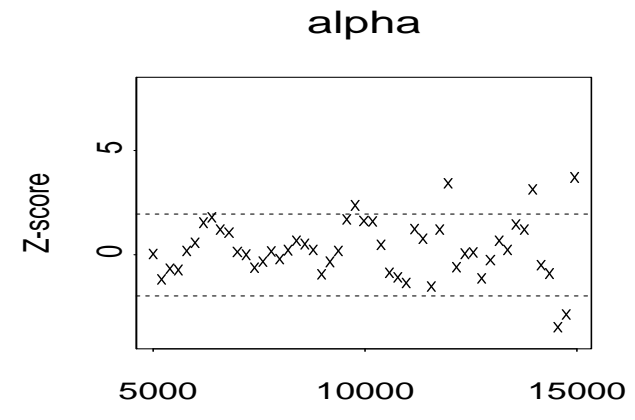

First iteration in segment

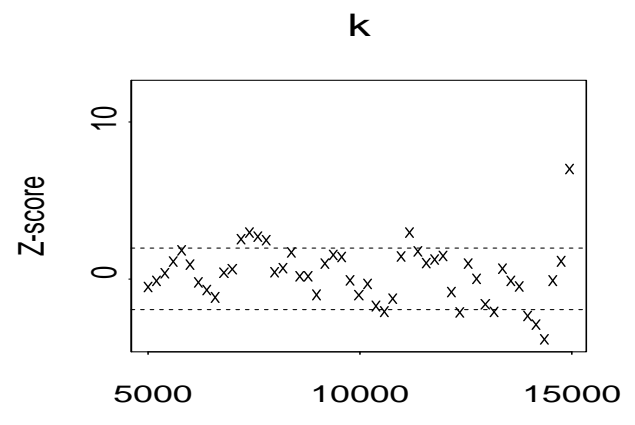

First iteration in segment

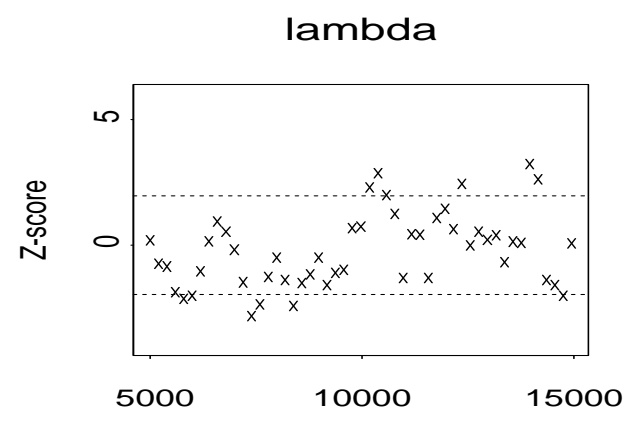

First iteration in segment

Figure 2: Geweke's diagnostic plots from WinBUGS output. 

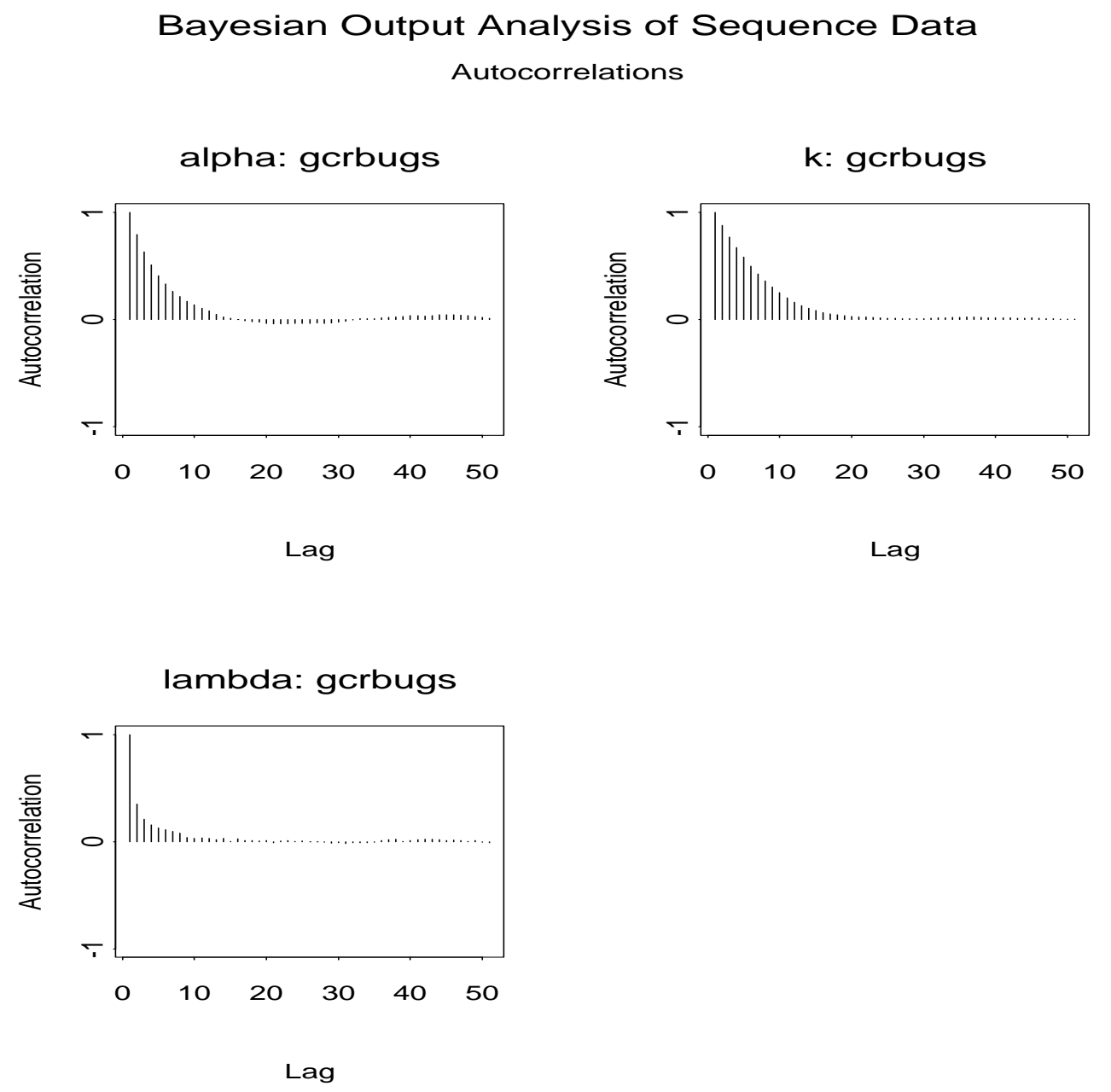

Figure 3: Auto-correlation function (ACF) plots from WinBUGS output based on all 10,000 samples. 

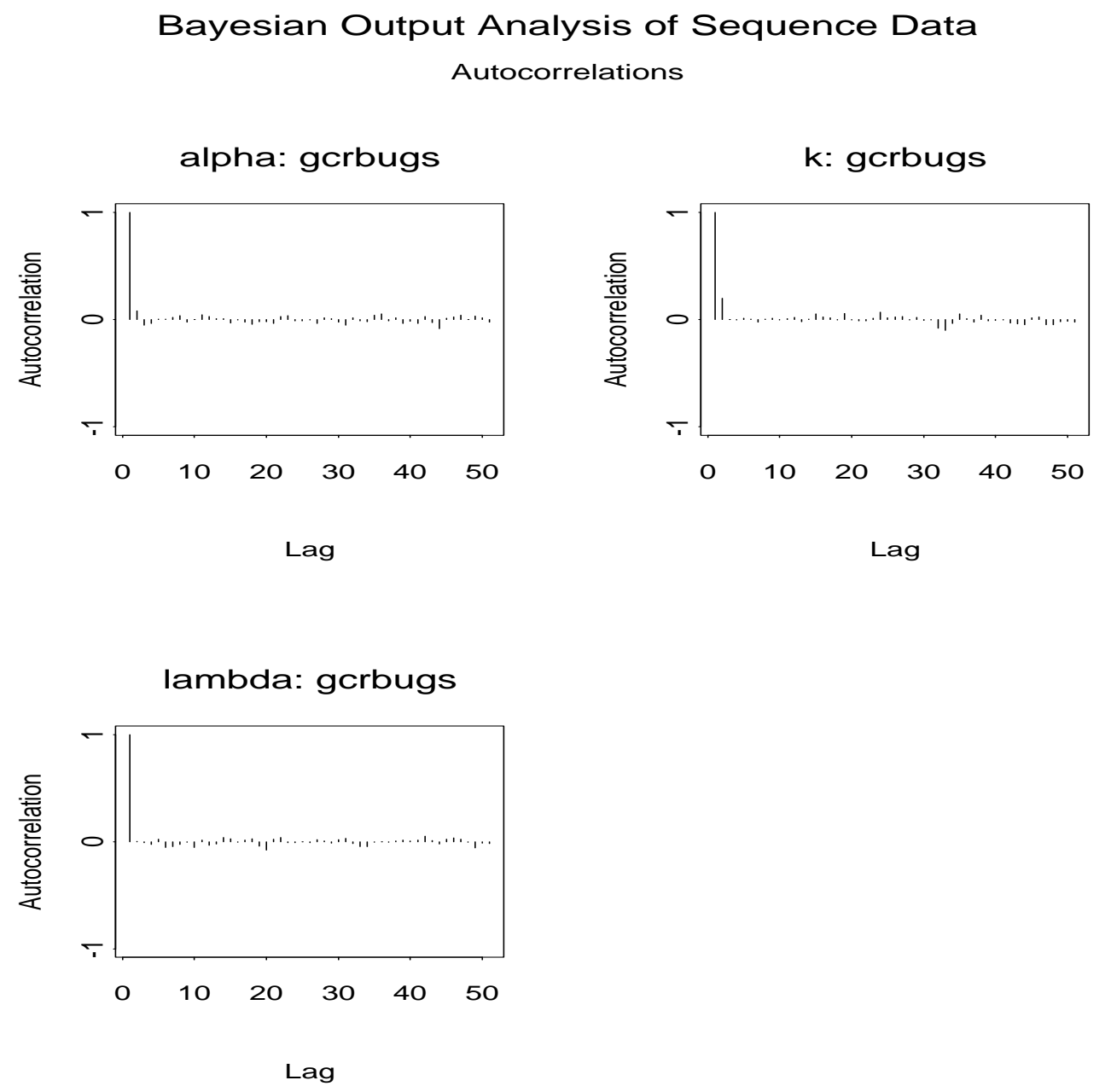

Figure 4: Auto-correlation function (ACF) plots from BUGS output, based on final 1000 samples discarding every 10th from the 10,000 samples. 